\title{
A Case of Right Sided Fixation of Sigmoid Colon in a Pediatric Patient with Late onset Hirshsprung's Disease
}

\author{
Shatrugna Kidambi ${ }^{1}$ and Ravikumar Hanumaiah ${ }^{1,2 *}$ \\ ${ }^{1}$ Columbia Asia Hospital, India \\ ${ }^{2}$ Department of Pediatric Radiology, SUNY Upstate Medical University, USA
}

*Corresponding author: Ravikumar Hanumaiah MD, SUNY Upstate Medical University, New York, USA, Email: h.ravi.k@gmail.com

\section{Case Report}

Volume 5 Issue 1

Received Date: May 24, 2021

Published Date: June 23, 2021

DOI: $10.23880 /$ crij- 16000187

\section{Abstract}

There are few reports in the scientific literature on the imaging features of right sided fixation of sigmoid colon in adult population, on Computed Tomography (CT). We present the imaging findings of right sided fixation of sigmoid colon and late onset Hirschsprung's disease in a 10 year old female and emphasize the need to consider this diagnosis in a child with chronic abdominal pain, constipation and a mass in the right abdomen.

Keywords: Right Sided Fixation of Sigmoid Colon; Late onset Hirschsprung's Disease; Malrotation; Computed Tomography

\section{Introduction}

Right sided fixation of sigmoid colon [1] is congenital colonic malposition. Generally, the sigmoid colon in adults is situated in the left lower quadrant with the sigmoid mesocolon attached to the left pelvic wall.

Hirschsprung's disease is a congenital functional obstruction of the colon caused by absence of ganglion cells in its distal portion, generally seen in the neonatal period. In cases of late onset, the diagnosis may be delayed due to presence of mild symptoms.

\section{Case report}

A 10 year old female presented with chronic right lower quadrant abdominal pain since 2 years and constipation. Sonography demonstrated heterogeneous hypoechoic mass in the right lower quadrant. Helical CT was performed after administration of positive oral and intravenous contrast during venous phase, $5 \mathrm{~mm}$ axial and coronal images were reconstructed. CT demonstrated the suspected right lower quadrant mass to be dilated sigmoid colon (Figure 1a). The rectum was not as dilated as the sigmoid colon (Figure 1a and $1 \mathrm{~b})$. There was associated colonic malposition with a right sided fixation of sigmoid colon.

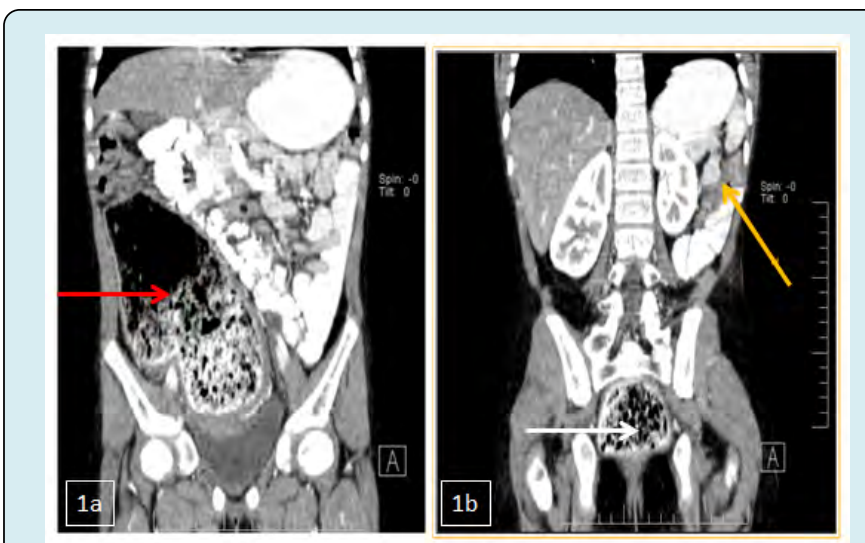

Figure 1a \& 1b: Coronal post contrast reformatted CT images of the abdomen and pelvis demonstrates a grossly dilated sigmoid colon (red arrow, Figure 1a) situated in the right side of abdomen, suggestive of right side fixation of the sigmoid colon. There is gradual narrowing of the rectum in the pelvis (white arrow, Figure $1 \mathrm{~b}$ ) with rectosigmoid ratio less than 1, suggestive of Hirshsprung's disease. The descending colon is collapsed (orange arrow, Figure 1b). 
The cecum and appendix were located high in the right upper quadrant (Figure 2a). The colon then followed a horizontal course (transverse colon) up to the splenic flexure in left upper quadrant. The descending colon originated in the left upper quadrant and then coursed horizontally across the midline anterior to the kidneys, aorta and Inferior vena cava (IVC), extending up to the right upper quadrant, inferior to the cecum. The descending colon then turned anteriorly and continued inferiorly as the dilated sigmoid colon on the right side of abdomen (Figure $2 \mathrm{~b}$ ).

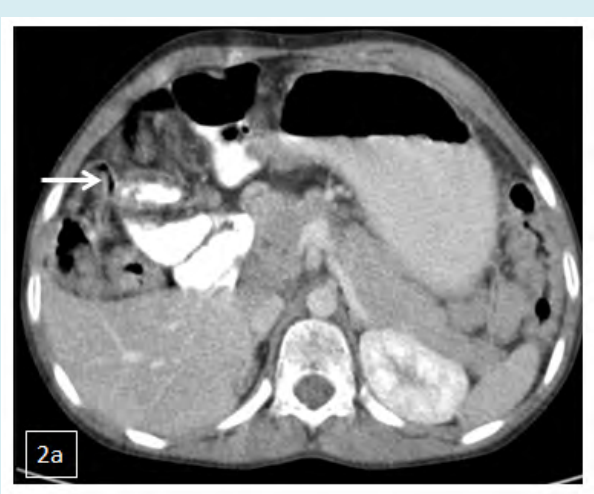

Figure 2a: Axial post contrast CT of the abdomen demonstrates the appendix (white arrow), cecum and ileocecal junction in the right upper quadrant, just inferior to the right lobe of liver.

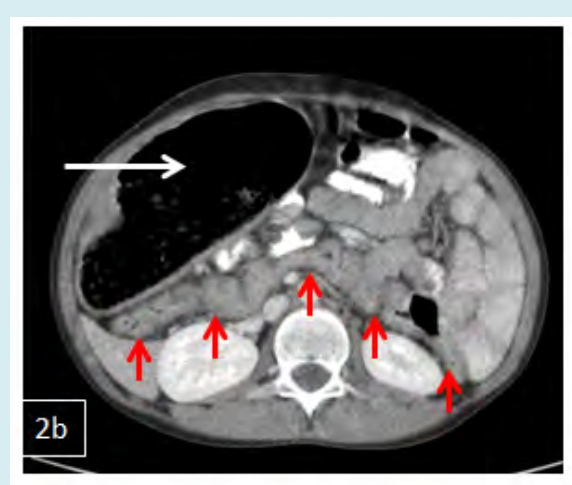

Figure 2b: Abnormal horizontal course of the descending colon across the midline, anterior to the kidneys, aorta and IVC - extending up to the right upper quadrant (red arrows). The descending colon then curves anteriorly and continues inferiorly as the sigmoid colon (white arrow, on the right side of abdomen) which is markedly distended with fecal material.

The sigmoid colon was markedly distended with fecal material. There was gradual narrowing of the rectum in the pelvis with rectosigmoid ratio less than 1 , suggestive of Hirshsprung's disease (Figure1a and 1b). This was a
Teleradiology case and contrast enema, manometry and biopsy confirmation were not available. The diagnosis was based on CT findings and clinical presentation. The most likely diagnosis was Hirshsprung's disease as other obstructive causes of bowel dilatation would cause a more proportionate dilatation of the entire colon rather than selective marked dilatation of the sigmoid colon seen in our case. The differential diagnosis includes redundant sigmoid colon with fecal loading.

The superior mesenteric vein (SMV) was anterior to the superior mesenteric artery (SMA) suggestive of malrotation (Figure 3). However, definitive diagnosis of midgut malrotation requires a fluoroscopy guided contrast upper gastrointestinal series.

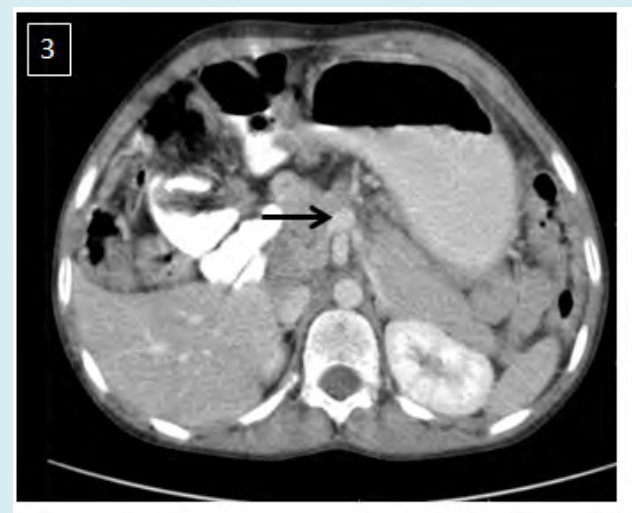

Figure 3: Axial contrast CT demonstrating superior mesenteric vein (black arrow) anterior to the superior mesenteric artery.

\section{Discussion}

During the 10th week of gestation, the distal midgut (Post arterial segment) returns to the peritoneal cavity and with its subsequent rotation, the colon assumes a normal configuration. On the right side, the development process comprises the formation of the hepatic flexure and descent of the cecum towards the right lower quadrant. On the left side, the hindgut is displaced laterally from a midline position by the jejunal loops with the formation of future splenic flexure and descending colon. The dorsal mesentery follows the colon in this process of rearrangement and completes its fusion to the posterior abdominal wall between the $4^{\text {th }}$ month and end of the $5^{\text {th }}$ month of gestation (Figure 4).

So far, very few cases of right side fixation of the sigmoid colon have been reported in adult patients, on CT [2]. Most of the cases reported are from cadaveric dissections. In one retrospective study of contrast enema examinations in children ( 2 months to 13 years) the position of sigmoid 


\section{Clinical Radiology \& Imaging Journal}

colon was in the right lower quadrant in $35.16 \%$ of cases, left lower quadrant in $36.26 \%$ of cases, midline in $13.19 \%$ of cases, and indeterminate in $15.38 \%$ of cases [3]. However, they recommended future studies with abdominal computed tomography and magnetic resonance imaging, in patients without suspected large bowel disease to evaluate the actual incidence of right sided fixation of sigmoid colon in children.

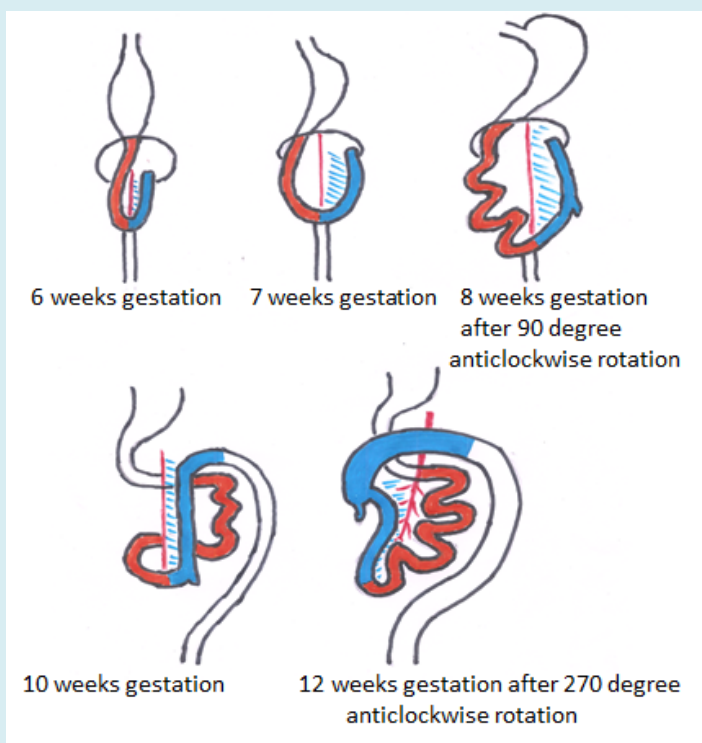

Figure 4: Chronology of normal intestinal rotation: The midgut loop undergoes three series of 90 degree rotations anti-clockwise around the SMA, resulting in a total of a 270 degree rotation anti-clockwise. The cranial limb goes from right to left of the SMA, whereas the caudal limb goes from left to right of the SMA, placing the small bowel in the center of the abdomen, the ascending colon and cecum to the right of the abdomen and the descending colon to the left.

In midgut malrotation, the cecum and the ascending colon float freely within the abdominal cavity, similar to the small bowel. Consequently, the empty space in the right side is occupied by the redundant sigmoid colon, and the fixation between the mesocolon of the sigmoid colon and pelvic wall on the right side may occur during the later period of the fetal life; and congenital colonic malposition occurs. The cause of right side fixation of the sigmoid colon may be a result of redundancy of sigmoid colon and/or midgut malrotation [2]. Also a small peritoneal band may be fixing the sigmoid colon to the ileocecal junction.

In our case, the cause is most likely to be midgut malrotation, given the CT findings of cecum in the right upper quadrant and SMV anterior to the SMA.
In patients with Hirschsprung's disease, both myenteric and submucosal plexuses are absent. The anus is invariably affected, and aganglionosis generally continues proximally for a variable distance. In the absence of enteric nervous system reflexes, control of the intestinal smooth muscle becomes extrinsic. This leads to an imbalance of smooth muscle contractility, uncoordinated peristalsis, and finally functional obstruction.

Radiographs of the neonatal abdomen with Hirschsprung's disease may show multiple loops of dilated small bowel with air-fluid levels suggestive of a distal bowel obstruction. An empty rectum is a common finding. The classic image is a dilated proximal colon with the aganglionic colon narrowing towards the rectum [4].

Hirschsprung's disease is diagnosed by means of contrast enema examination, which can show the presence of a transition zone, irregular contractions, mucosal irregularity, and delayed evacuation of contrast material, among other findings. Contrast enemas should be avoided in patients with enterocolitis because of the risk of perforation [5]. The definitive diagnosis is by rectal biopsy.

\section{Conclusion}

The combination of right sided fixation of sigmoid colon associated with late onset Hirschsprung's disease can lead to the clinical presentation of chronic constipation and a palpable mass on the right side of abdomen. CT scan can rule out a neoplasm as well as confirm both these entities.

The knowledge of right sided fixation of sigmoid colon is necessary for planning colonoscopy procedures and colonic surgeries/ interventions.

\section{References}

1. Chandrika Gurulingappa Teli, Gnanagurudasan (2012) Right Sided Sigmoid Colon-Rare Case. Int J Biol Med Res 3(2): 1842-1844.

2. Choi NG, Moon OI, Kim JH, Sharon Lim, Sung Chul Lim, et al. (2013) Right side fixation of sigmoid colon with a hepato-sigmoidocolic fistula in patient with hepatocellular carcinoma and midgut malrotation. J Korean Surg Soc 84(4): 256-260.

3. Saxena AK, Sodhi KS, Trumani S, Mumtaz H A, Narasmha Rao KL, et al. (2011) Position of a sigmoid colon in right iliac fossa in children: A retrospective study. J. Indian Assoc Pediatr Surg 16(3): 93-96. 
4. Amiel J, Sproat-Emison E, Garcia-Barcelo M, Lantieri F, Burzynski G, et al. (2008) Hirschsprung disease, associated syndromes and genetics: a review. J Med Genet 45(1): 1-14.
5. Stranzinger E, Dipietro MA, Teitelbaum DH, Strouse PJ (2008) Imaging of total colonic Hirschsprung disease. Pediatr Radiol 38(11): 1162-1170. 